\title{
Simultaneous measurements of thermal conductivity and diffusivity of $\operatorname{Se}_{80} \mathrm{Te}_{20-x} \mathrm{In}_{x}(x=2,4,6$ and 10) chalcogenide glasses at room temperature
}

\author{
N S SAXENA*, MOUSA M A IMRAN and KEDAR SINGH \\ Department of Physics, University of Rajasthan, Jaipur 302 004, India
}

MS received 17 July 2001; revised 14 February 2002

\begin{abstract}
Measurements of thermal conductivity and thermal diffusivity of twin pellets of $\operatorname{Se}_{80} \mathrm{Te}_{20-x} \mathbf{I n}_{x}$ $(x=2,4,6$ and 10) glasses, prepared under a load of 5 tons were carried out at room temperature using transient plane source (TPS) technique. The measured values of both thermal conductivity and diffusivity were used to determine the specific heat per unit volume of the said materials in the composition range of investigation. Results indicated that both the values of thermal conductivity and thermal diffusivity increased with the addition of indium at the cost of tellurium whereas the specific heat remained almost constant. This compositional dependence behaviour of the thermal conductivity and diffusivity has been explained in terms of the iono-covalent type of bond which In makes with Se as it is incorporated in the Se-Te glass.
\end{abstract}

Keywords. Chalcogenide glasses; thermal conductivity; specific heat; iono-covalent bond.

\section{Introduction}

Chalcogenide glasses are interesting materials because of their technological applications (Tanaka 1989) and commercial importance (Seddon 1995). The addition of indium as third element at different percentages in Se-Te binary chalcogenide glasses produces stability (Pradeep et al 1996) in these glasses. The effect of the element as an additive to binary glasses has been extensively studied (Agrawal et al 1991). In the present work, measurements of thermal conductivity, thermal diffusivity and specific heat of these glasses have been carried out at room temperature. The transient plane source (TPS) technique (Gustaffson 1991) was used for these measurements.

\section{Transient plane source theory}

The TPS technique has proved to be a precise and convenient method for measuring the thermal transport properties of electrically insulating materials. The TPS method consists of an electrically conducting pattern (figure 1) in the form of a bifilar spiral, which also serves as a sensor of the temperature increase in the sample. In figure $1 \mathrm{~K}-4521$ is the design number of the sensor and $\mathrm{K}$ stands for kapton. The sensor is sandwiched between the thin insulating layers of kapton. Assuming the conductive pattern to be in the $\mathrm{Y}-\mathrm{Z}$ plane of a coordinate system, the rise in the temperature at a point $\mathrm{Y}-\mathrm{Z}$ at time $t$ due to an

\footnotetext{
*Author for correspondence
}

output power per unit area, $Q$, is given by (Carlaw and Jagar 1959)

$$
\begin{aligned}
& \Delta T(y, z, \tau)=\frac{1}{4 \pi^{3 / 2} a \lambda} \int_{0}^{\tau} \frac{\mathrm{d} \sigma}{\sigma^{2}} \int_{A} \mathrm{~d} y^{\prime} \mathrm{d} z^{\prime} \\
& \times^{\prime} Q\left(y^{\prime} z^{\prime} t-\frac{\sigma^{2} a^{2}}{\kappa}\right) \exp \left[\frac{-\left(y-y^{\prime}\right)^{2}-\left(z-z^{\prime}\right)^{2}}{4 \sigma^{2} a^{2}}\right],
\end{aligned}
$$

where $k\left(t-t^{\prime}\right)=\sigma^{2} a^{2}, \theta=a^{2} / k$, and $\tau=[t / \theta]^{1 / 2}, a$ is the radius of the hot disc which gives a measurement of the overall size of resistive pattern and $\theta$ is known as the characteristic time. $\sigma$ is a constant variable and $\lambda$ the thermal conductivity in the units of $\mathrm{W} / \mathrm{mK}$ and $k$ the thermal diffusivity in unit of $\mathrm{m}^{2} / \mathrm{s}$. The temperature increase, $\Delta T(y, z, \tau)$, because of flow of current through the sensor gives rise to a change in the electrical resistance, $\Delta R(t)$, which is given as

$$
\Delta R(t)=\alpha R_{0} \overline{\Delta T(\tau)}
$$

where $R_{0}$ is resistance of TPS element before the transient recording was initiated, $\alpha$ the temperature coefficient of resistance (TCR) and $\overline{\Delta T(\tau)}$ the mean value of the time dependent temperature increase of the TPS element. $\overline{\Delta T(\tau)}$ is calculated by averaging the increase in temperature of TPS element over the sampling time because the concentric ring sources in TPS element have different radii and are placed at different temperatures during the transient recording (Carlaw and Jagar 1959) 


$$
\begin{aligned}
\overline{\Delta T(\tau)} & =\frac{P_{0}}{\pi^{3 / 2} a \lambda} D_{\mathrm{s}}(\tau), \\
D_{\mathrm{s}}(\tau)= & {[m(m+1)]^{-2} \int_{0}^{\tau} \frac{\mathrm{d} \sigma}{\sigma^{2}} } \\
& \times\left[\sum_{l=1}^{m} 1\left\{\sum_{k=1}^{m} k e \frac{-\left(l^{2}+k^{2}\right)}{2 \sigma^{2} m^{2}} L_{0}\left(\frac{1 k}{2 \sigma^{2} m^{2}}\right)\right\}\right],
\end{aligned}
$$

where $P_{0}$ is the total output power, and $L_{0}$ the modified Bessel function. To record the potential difference variations, which normally are of the order of a few millivolts during the transient recording, a simple bridge arrangement as shown in figure 2 has been used. If we assume that the resistance increase will cause a potential difference variation, $\Delta U(t)$, measured by the voltmeter in the bridge, the analysis of the bridge indicates that

$$
\Delta E(t)=\frac{R_{\mathrm{s}}}{R_{\mathrm{s}}+R_{0}} I_{0} \Delta R(t)=\frac{R_{\mathrm{s}}}{\left(R_{\mathrm{s}}+R_{0}\right)} \frac{I_{0} \alpha R_{0} P_{0}}{\pi^{3 / 2} a \lambda} D_{\mathrm{s}}(\tau),
$$

where

$$
\Delta E(t)=\Delta U(t)[1-C \cdot \Delta U(t)]^{-1},
$$

and

$$
C=\frac{1}{R_{\mathrm{s}} I_{0}\left[1+\frac{\gamma R_{\mathrm{p}}}{\gamma\left(R_{\mathrm{s}}+R_{0}\right)+R_{\mathrm{p}}}\right]} .
$$

The definition of various resistances is found in figure 2 . $R_{\mathrm{s}}$ is a standard resistance with a current rating that is much higher than $I_{0}$, which is $a$, the initial heating current through the arm of the bridge containing the TPSelement.

\section{Material preparation}

High purity $(99.999 \%) \mathrm{Se}$, Te and In in appropriate atomic percentages were weighed into a quartz glass ampoule (length, $5 \mathrm{~cm}$ and internal diameter, $8 \mathrm{~mm}$ ). The contents of the ampoule $(5 \mathrm{~g})$ were sealed into a vacuum of $10^{-6}$ Torr and heated in a furnace where temperature

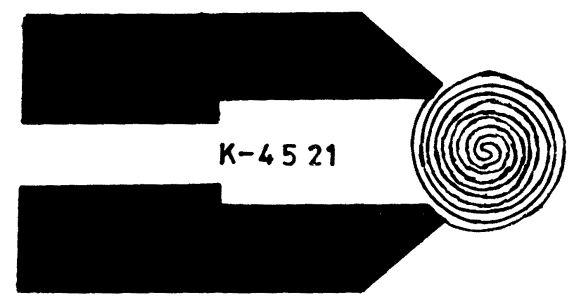

Figure 1. Schematic diagram of TPS sensor. was raised at a rate of 3-4 K per min up to $925 \mathrm{~K}$ and kept around that temperature for $7-8 \mathrm{~h}$ to ensure the homogeneity of the samples. The molten samples were then rapidly quenched in ice cooled water. Samples obtained by quenching were in the form of glasses. The glassy nature has been confirmed through X-ray diffraction. These bulk glasses were then crushed to fine powders by grinding process. Pellets of thickness, $1 \mathrm{~mm}$ and diameter, $12 \mathrm{~mm}$ were prepared by a pressure machine at a pressure of 5 tons.

\section{Experimental}

The measurements reported in this paper were performed with a TPS element of the type shown in figure 1. TPS element is made of $10 \mu \mathrm{m}$ thick nickel foil with an insulating layer made of $50 \mu \mathrm{m}$ thick kapton, on each side of the metal pattern. Evaluation of these measurements was performed in a way that was outlined by Gustaffson et al (1986). In experiments with insulating layers of such thickness, it is necessary to ignore the voltage recorded during the first few seconds because of the influence of the insulating layers. However, owing to the size of the heated area of the TPS element, the characteristic time of the experiment is so long that it is possible to ignore a few seconds of recorded potential difference values.

No influence could be recorded from electrical connections, which are shown in figure 2 . These connecting leads had the same thickness as the metal pattern of the TPS element. Each TPS element had a resistance at room temperature of about $3.26 \Omega$ and a TCR of around $4.6 \times 10^{-3} \mathrm{~K}^{-1}$.

An important aspect of design of any TPS element is that the pattern should be such that as large a part of the "hot" area as possible should be covered by the electrically conducting pattern, as long as there is insulation between different parts of the pattern. This is particularly important when insulating layers are covering the conduction pattern and the surface(s) of the sample. It should

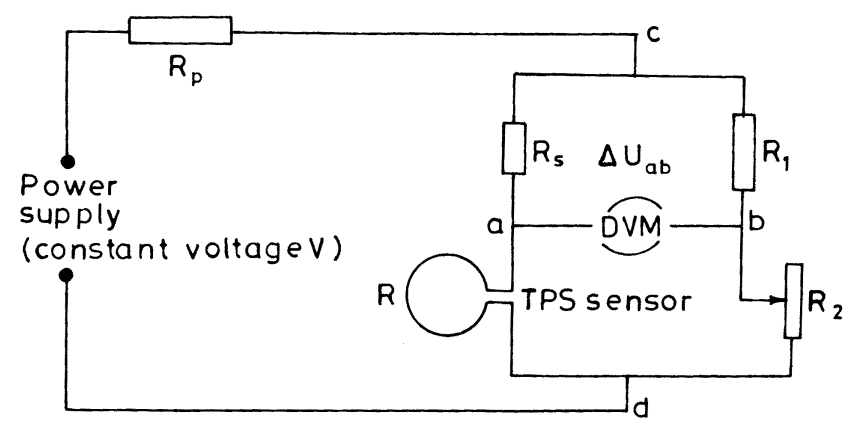

Figure 2. Schematic diagram of electrical circuit used for simultaneous measurement of thermal conductivity and thermal diffusivity. 
be noted that the temperature difference across the insulating layer can, after a short initial transient, be considered constant.

The samples are in the form of pellets of $12 \mathrm{~mm}$ diameter and $1 \mathrm{~mm}$ thickness, and the surfaces of these pellets are smooth so as to ensure perfect thermal contact between the samples and the heating elements, as the TPS sensor is sandwiched between the two pellets of sample material in the sample holders using pressure contacts (figure 3 ). The change in the voltage was recor-

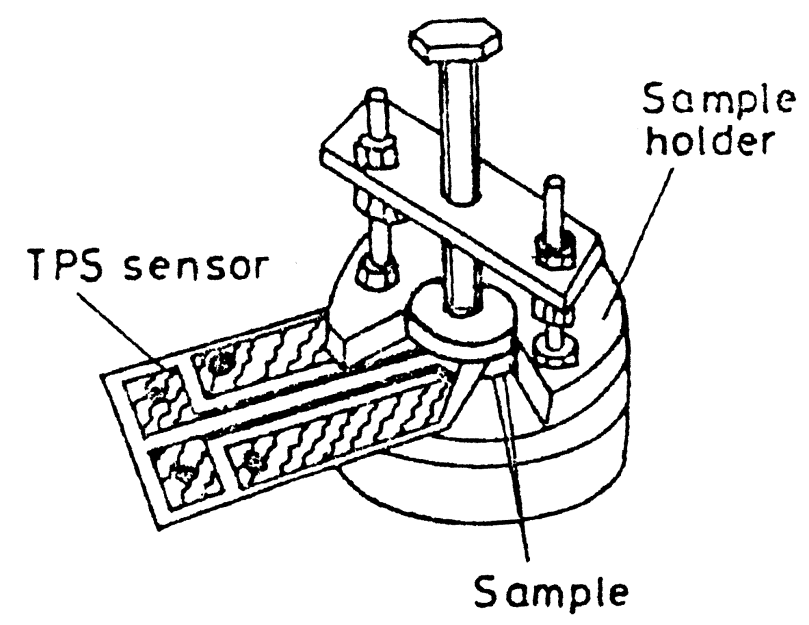

Figure 3. Sample holder diagram with TPS sensor. ded with a digital voltmeter, which was online to the personal computer. The power output to the sample was adjusted according to the nature of the sample material and was, in most cases, in the range 0.06$0.16 \mathrm{~W} / \mathrm{cm}^{2}$.

\section{Results and discussion}

Simultaneous measurements of effective thermal conductivity and effective thermal diffusivity of pellets of $\mathrm{Se}_{80} \mathrm{Te}_{20-x} \operatorname{In}_{x} \quad(x=2,4,6$ and 10) glasses, compacted under a load of 5 tons, were carried out at room temperature using TPS technique. These measured values of effective thermal conductivities and diffusivities were used to obtain the values of specific heat per unit volume of these glasses at different compositions of Te and In. The results of these measurements are given in table 1 . In order to show the variation of effective thermal conductivity $\left(\lambda_{\mathrm{e}}\right)$, effective thermal diffusivity $\left(k_{\mathrm{e}}\right)$ and specific heat per unit volume $\left(\rho C_{\mathrm{p}}\right)$ with the composition $(x)$ of indium, the results have been plotted in figures $4-6$, respectively. It can be observed from figures $4-5$, that the effective thermal conductivity and effective thermal diffusivity of the glasses increased slightly with the increase of composition of indium in the Se-Te-In glass. Slight increases in the effective thermal conductivity and effective thermal diffusivity could be explained by considering the structural changes due to the introduction of more and more indium atoms. The structure of the

Table 1. Thermal conductivity, diffusivity and specific heat per unit volume of the $\operatorname{Se}_{80} \operatorname{Te}_{20-x} \operatorname{In}_{x}(x=2,4,6$ and 10$)$ chalcogenide glasses.

\begin{tabular}{|c|c|c|c|c|c|}
\hline Sample name & Experiment & $\begin{array}{c}\text { Thermal } \\
\text { conductivity, } \lambda_{\mathrm{e}} \\
\text { (Watt } / \mathrm{m}-\mathrm{K} \text { ) } \\
\text { by TPS }\end{array}$ & $\begin{array}{l}\text { Thermal } \\
\text { diffusivity, } k_{\mathrm{e}} \\
\left(\mathrm{mm}^{2} / \mathrm{s}\right) \\
\text { by TPS }\end{array}$ & $\begin{array}{l}\text { Specific heat per } \\
\text { unit volume, } \rho C_{\mathrm{p}} \\
\left(\mathrm{MJ} / \mathrm{m}^{3} \mathrm{~K}\right) \\
\text { by TPS }\end{array}$ & $\begin{array}{c}\text { Specific heat per } \\
\text { unit volume, } \rho C_{\mathrm{p}} \\
\left(\mathrm{MJ} / \mathrm{m}^{3} \mathrm{~K}\right) \\
\text { by DSC }\end{array}$ \\
\hline \multirow[t]{4}{*}{$\mathrm{Se}_{80} \mathrm{Te}_{18} \mathrm{In}_{2}$} & Expt. I & $0 \cdot 165$ & $0 \cdot 178$ & 0.927 & \\
\hline & Expt. II & $0 \cdot 167$ & $0 \cdot 176$ & 0.948 & \\
\hline & Expt. III & $0 \cdot 164$ & $0 \cdot 178$ & 0.947 & \\
\hline & Av. & $0 \cdot 165$ & $0 \cdot 176$ & 0.940 & $3 \cdot 09$ \\
\hline \multirow[t]{4}{*}{$\mathrm{Se}_{80} \mathrm{Te}_{16} \mathrm{In}_{4}$} & Expt. I & $0 \cdot 179$ & $0 \cdot 180$ & $0 \cdot 991$ & \\
\hline & Expt. II & $0 \cdot 175$ & $0 \cdot 182$ & 0.968 & \\
\hline & Expt. III & $0 \cdot 174$ & $0 \cdot 177$ & 0.981 & \\
\hline & Av. & $0 \cdot 176$ & $0 \cdot 180$ & $0 \cdot 980$ & $3 \cdot 44$ \\
\hline \multirow[t]{4}{*}{$\mathrm{Se}_{80} \mathrm{Te}_{14} \mathrm{In}_{6}$} & Expt. I & $0 \cdot 186$ & $0 \cdot 190$ & 0.979 & \\
\hline & Expt. II & $0 \cdot 183$ & $0 \cdot 186$ & 0.983 & \\
\hline & Expt. III & $0 \cdot 182$ & $0 \cdot 187$ & 0.969 & \\
\hline & Av. & $0 \cdot 183$ & $0 \cdot 187$ & 0.977 & $2 \cdot 755$ \\
\hline \multirow[t]{4}{*}{$\mathrm{Se}_{80} \mathrm{Te}_{10} \mathrm{In}_{10}$} & Expt. I & $0 \cdot 191$ & $0 \cdot 200$ & 0.909 & \\
\hline & Expt. II & $0 \cdot 197$ & $0 \cdot 197$ & 0.954 & \\
\hline & Expt. III & $0 \cdot 194$ & $0 \cdot 198$ & 0.950 & \\
\hline & Av. & $0 \cdot 194$ & $0 \cdot 199$ & 0.933 & $1 \cdot 377$ \\
\hline
\end{tabular}


Se-Te system prepared by melt quenching is regarded (Lucovsky 1987) as a mixture of $\mathrm{Se}_{8}$ rings, $\mathrm{Se}_{6} \mathrm{Te}_{2}$ rings and $\mathrm{Se}-\mathrm{Te}$ copolymer chains. In the present case, the addition of In is at the cost of Te concentration. Indium makes iono-covalent bonds with $\mathrm{Se}$ and is probably dissolved in the Se-chain, making the system more and more thermally stable with the increase of indium in the binary Se-Te system. Thermal stability of the chalcogenide glasses is directly related to the thermal conductivity and thermal diffusivity. These iono-covalent bonds having high dissociation energy offer more conductive path in the system and hence provide an easier heat flow from one point to another in the alloy. The effect of formation of iono covalent bond in these glasses is also reflected in several kinetic parameters (Imran et al 2001) during their thermal analysis using differential scanning calorimetry (DSC).

Specific heat per unit volume $\left(\rho C_{\mathrm{p}}\right)$ as obtained from the experimentally measured values of thermal conductivity and thermal diffusivity, is shown in table 1 , for different compositions of indium. Figure 6 shows the variation of specific heat per unit volume with the composition $(x)$ of indium in Se-Te-In alloy. It can be observed that the variation in specific heat is very small and for all practical purposes it can be treated as constant. The values of specific heat (Imran et al 2000)

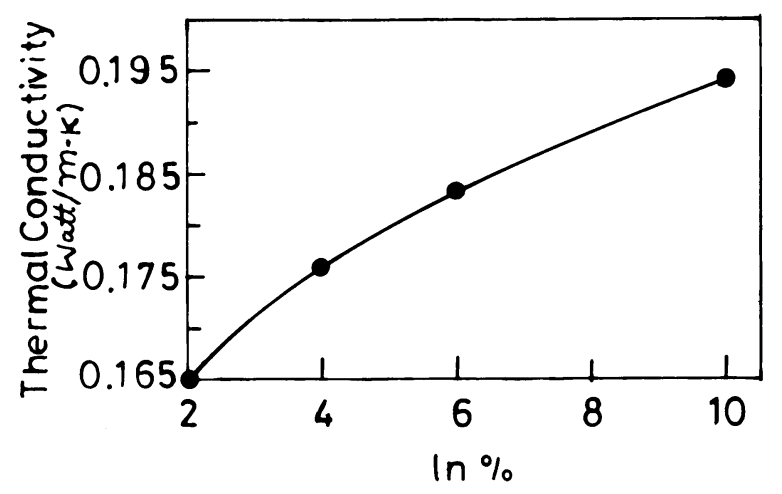

Figure 4. Thermal conductivity vs indium percentage.

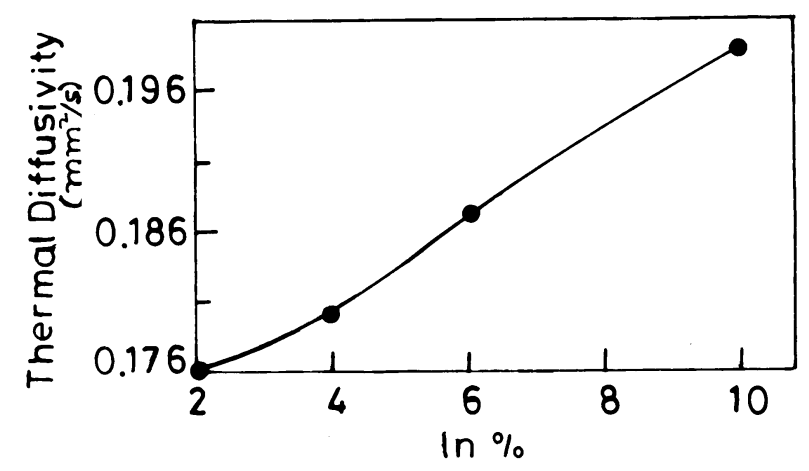

Figure 5. Thermal diffusivity vs indium percentage. from DSC at different temperatures are also given in the same table. The trend of variation of specific heat as obtained from the two separate experiments is similar. However, there are differences of magnitudes of specific heats per unit volume for different compositions in two cases. The values of specific heat per unit volume as derived in TPS experiment also agree with the Dulong Pettits law at room temperature whereas, in the DSC experiment the values even at room temperature do not agree with that limit. This might be due to slightly different configuration (structure) of the samples used in the two experiments. The higher value of the porosity of the material (in the form of pressed pellets) used in TPS gives the lower values of thermal conductivity and diffusivity, as compared to the solid bulk of the material taken in DSC, and hence in the specific heat per unit volume. However, these values of $\rho C_{p}$ are more reliable than the values of $\rho C_{\mathrm{p}}$ obtained from the DSC experiment.

Slightly lower values of specific heat at higher composition of indium are due to the non-availability of the large number of degrees of freedom in the alloy, which could absorb heat energy. The degrees of freedom available for absorbing heat energy will be lesser and lesser as the indium is added, because of the overall increased thermal stability of system. Measurement of these properties, i.e. thermal conductivity and thermal diffusivity, has been carried out at room temperature. Temperature dependence of thermal properties has been tried in case of $\mathrm{Se}-\mathrm{Te}-\mathrm{Sb}$ in our laboratory. It was found that as the alloy has been kept at different temperatures over the room temperature its structure changes continuously and transformations are shown at glass transition and crystallization temperatures. Due to this behaviour of material, it had been very difficult to take measurements (atleast three experiments at a particular temperature) which were reproducible within the experimental error. However, the study on temperature dependence of thermal conductivity and thermal diffusivity on samples annealed at different temperatures and for different times is in progress.

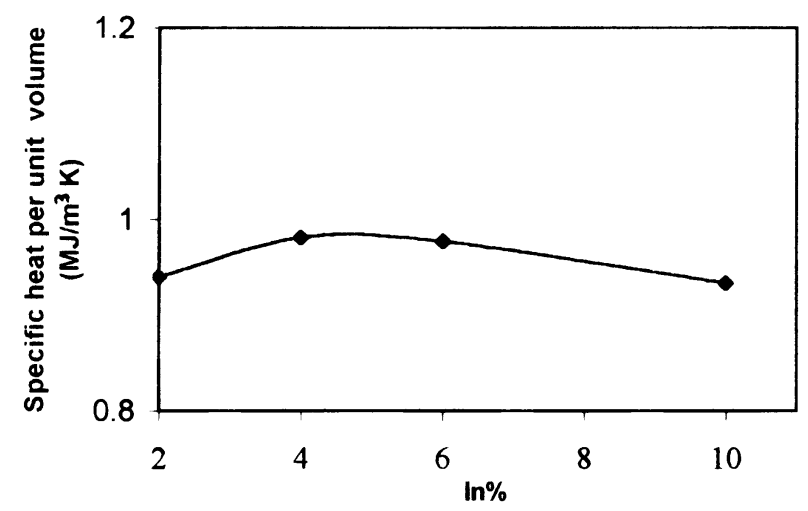

Figure 6. Specific heat per unit volume vs indium percentage. 


\section{Conclusion}

Lower values of effective thermal conductivities and effective thermal diffusivities of the samples at given composition of indium under study are suggestive of the fact that the thermophysical properties can show large variation at the higher composition of indium for a binary glass of Se-In and not the ternary glasses.

\section{References}

Agrawal P, Goil S, Rai J P S and Kumar A 1991 Phys. Status Solidi (a)127 363
Carlaw H S and Jaeger J C 1959 Conduction of heat in solids (New York: Oxford Univ. Press) p. 510

Gustaffson S E 1991 Rev. Sci. Instrum. 62197

Gustaffson S E, Karawaki E and Chohan M A 1986 J. Phys. D. Appl. Phys. 19727

Imran Mousa M A, Bhandari D and Saxena N S 2000 Mater. Sci. Eng. A292 56

Imran Mousa M A, Bhandari D and Saxena N S $2001 \mathrm{~J}$. Therm. Anal. \& Calor. $\mathbf{6 5} 257$

Lucovsky G J 1987 Non Cryst. Solids $97 / 983950$

Pradeep P, Saxena N S, Saksena M P and Kumar A 1996 Phys. Status Solidi 156222

Seddon A B 1995 J. Non-Cryst. Solids 18444

Tanaka K 1989 Phys. Rev. B39 1270 